\title{
Joint and Individual Walking in an Immersive Collaborative Virtual Environment
}

\author{
Stephan Streuber, Astros Chatziastros, Stephan de la Rosa, \& Heinrich H. Bülthoff \\ - Max Planck Institute for Biological Cybernetics -
}

\section{Questions:}

Humans cooperate with other humans on a daily basis, for example, when carrying a stretcher. Is our cooperative behavior guided by immediate sensory feedback or by our knowledge about the task (mental task representation)? We investigated this question in a stretcher carrying task.

\section{Logic:}

When two people are carrying a stretcher, one person leads $(L)$ while the other person follows (F). Importantly, when carrying the stretcher around a corner, only the follower has visual information about the distance between the stretcher and the corner (see Figure 1). If the leader does not use task knowledge to guide his behavior, (s)he should walk around the corner as if (s)he were walking alone. As a result the follower's task would be to guarantee the correct maneuver of the stretcher around the corner. Hence leader and follower should have different walking behavior. On the other hand if the leader uses knowledge about the task to guide his behavior, the leader would adjust his behavior in such a way that it supports that the stretcher does not hit the wall. This behavior should result in similar walking behavior of leader and follower. Consequently the comparison of walking behavior between leader and follower is indicative of whether the people use knowledge about a task in collaborative situations.

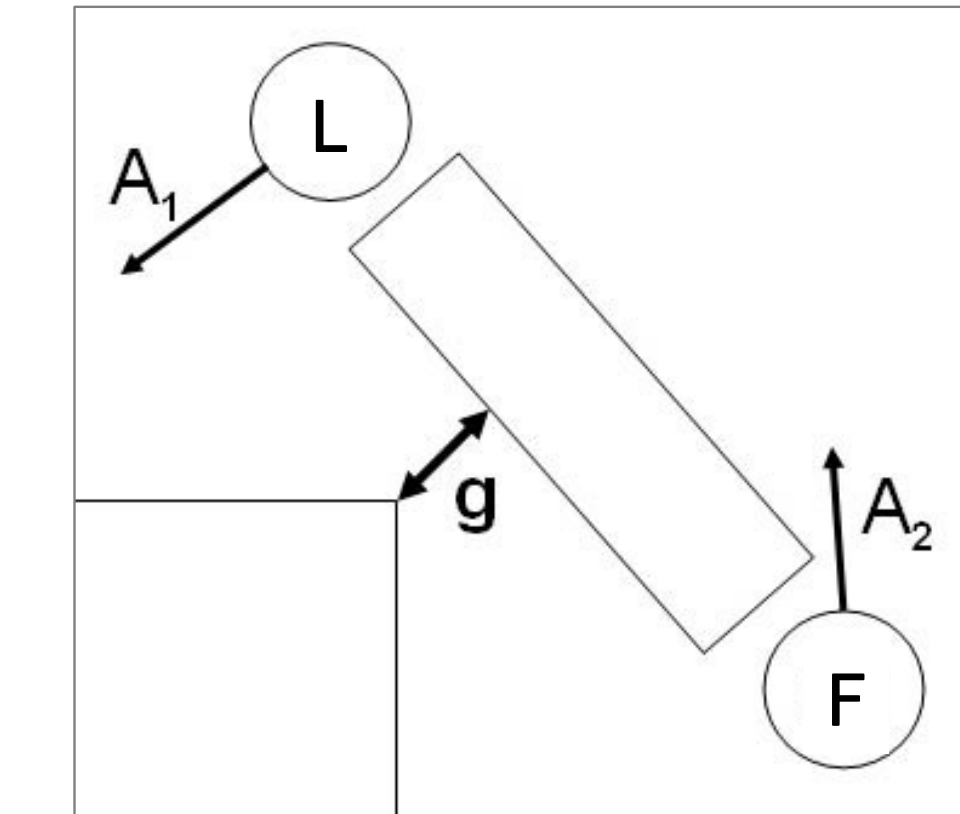

Figure 1: Schematic outline of carrying a stretcher around a corner. The leader (L) cannot see the follower (F). Arrows indicate the walking direction $g>0$ to direction. $g>0$ to

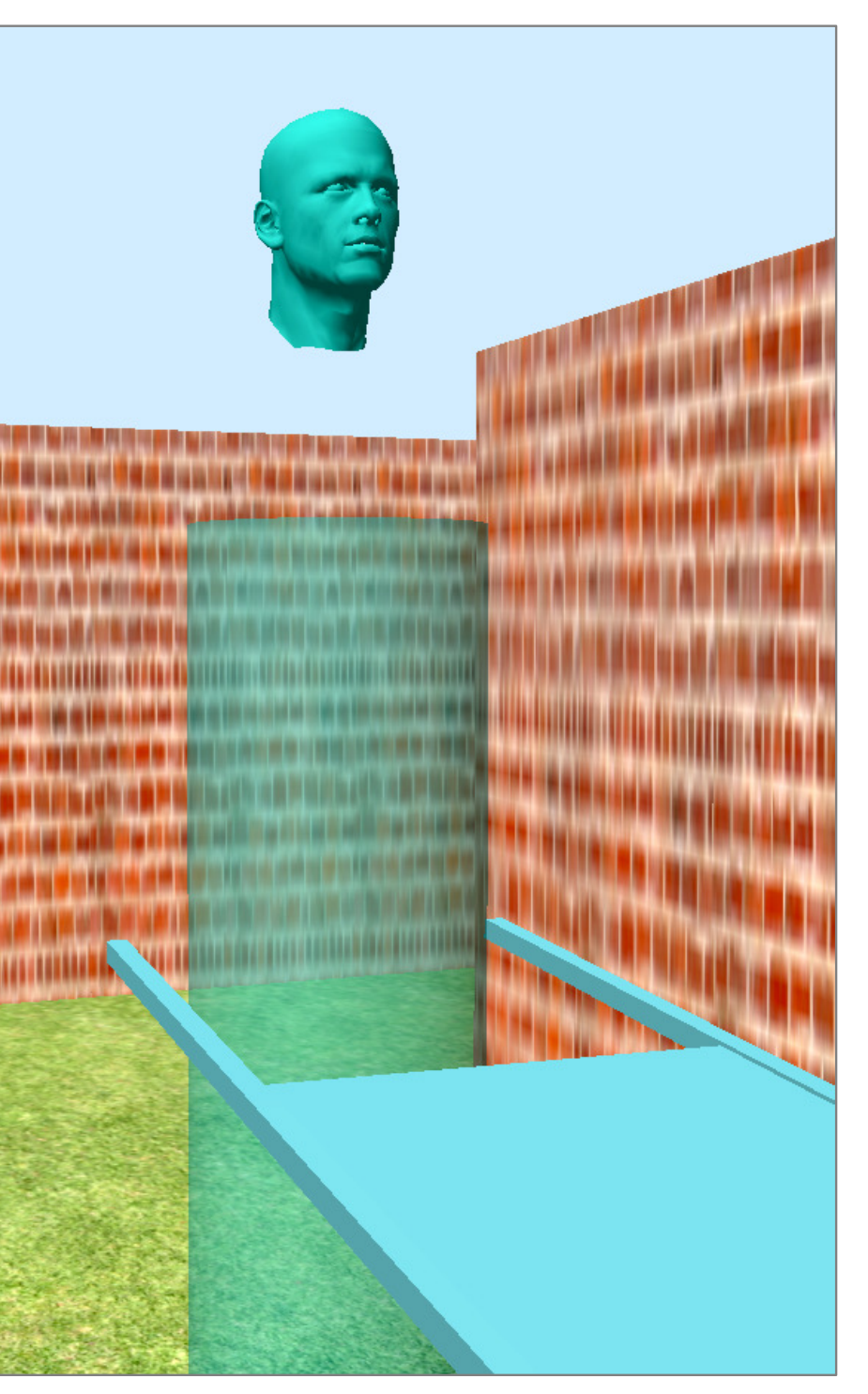

Figure 2: Example display of the virtual environment including the stretcher, the other person, and the maze from a $3^{\text {rd }}$ person perspective (not possible during the experiment) stretcher and the

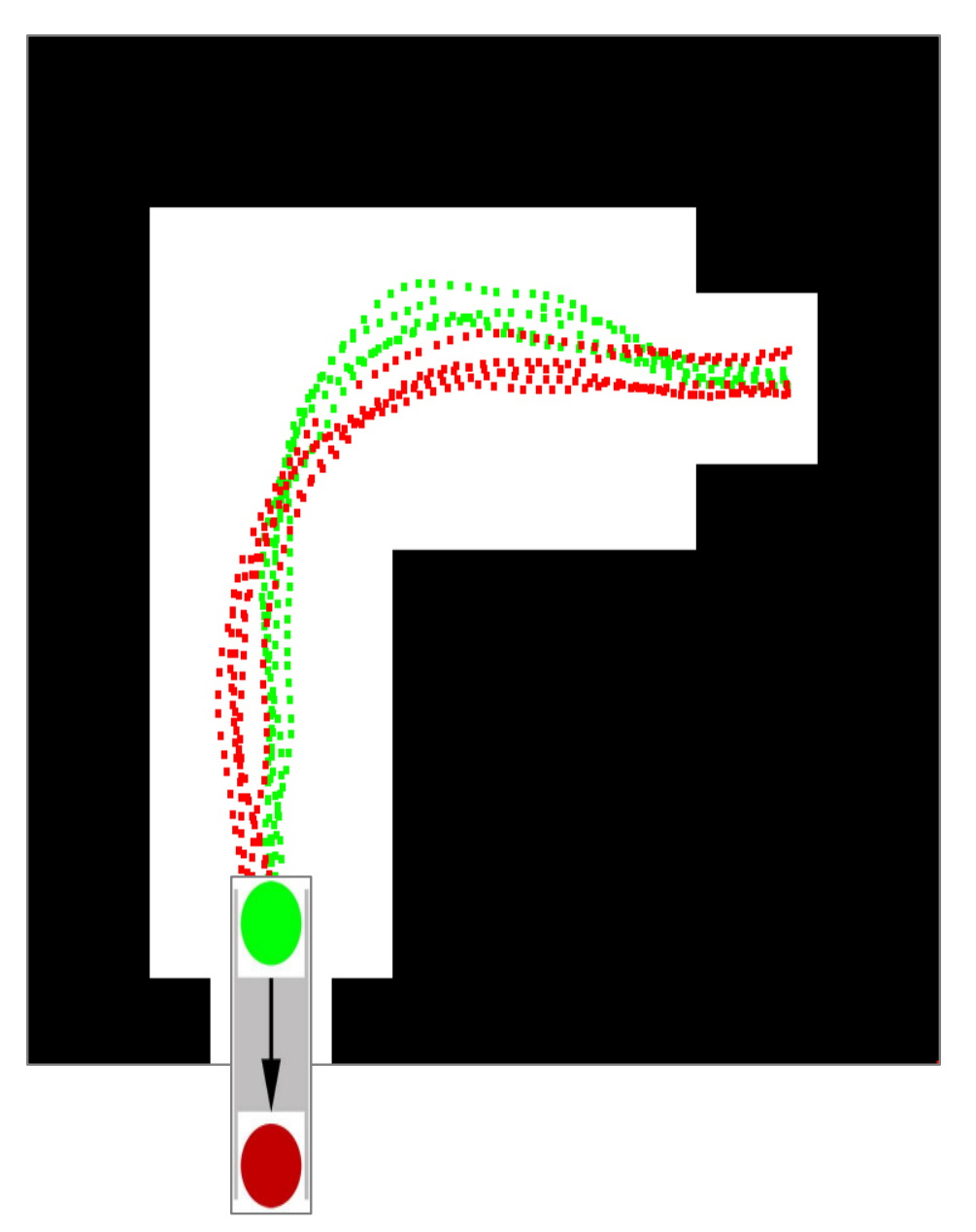

Figure 3: Walking trajectories of two participants carning the stretcher around a corner. Leader is in green and follower in red.

\section{Experiment 1:}

Twelve participants $(n=12)$ walked through a virtual maze either individually (=Individual Condition) or jointly connected via the stretcher (=Joint Condition) (see also Figure 2). Walking behavior was assessed in terms of path length and power law.

Results:
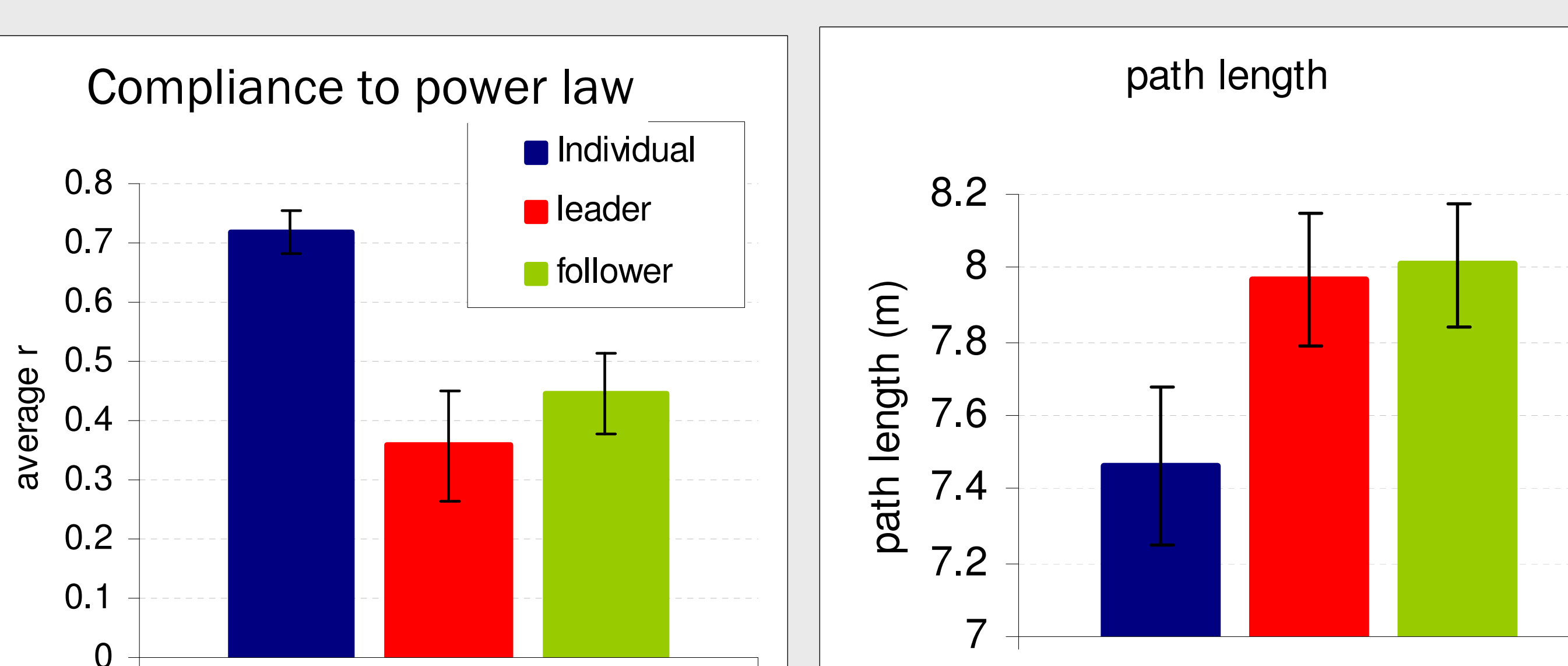

Conclusions:

- Participants had similar walking behavior. The leaders walking behavior differed significantly from the individual walking behavior.

- Results support the idea that the leader used task knowledge.

Alternative explanation: The follower guided the leader by pushing the stretcher (haptic communication). Experiment 2 assessed this alternative.

\section{Experiment 2:}

Task and Conditions:

In two conditions we tested subjects $(n=20)$ walking either jointly connected via the physical stretcher (=Joint Condition) or jointly connected via virtual stretcher (=Virtual Condition). We expect to observe difference between these two condition if the follower guided the leader by pushing/pulling the physical stretcher.

\section{Results and Conclusions}

- Participants shared had similar walking behavior in both conditions.

- These results further support the idea that task knowledge guides the leader's behavior.

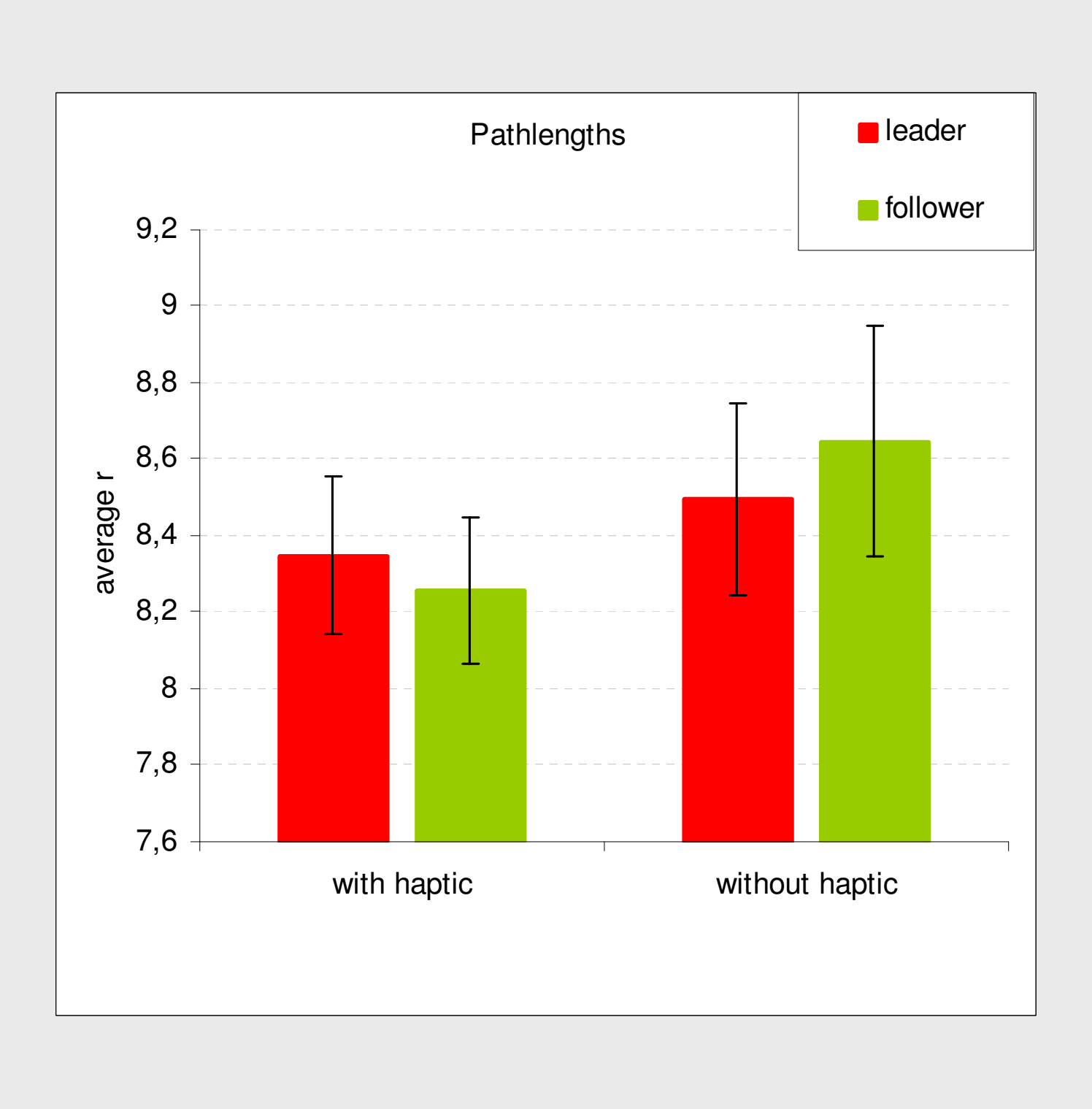

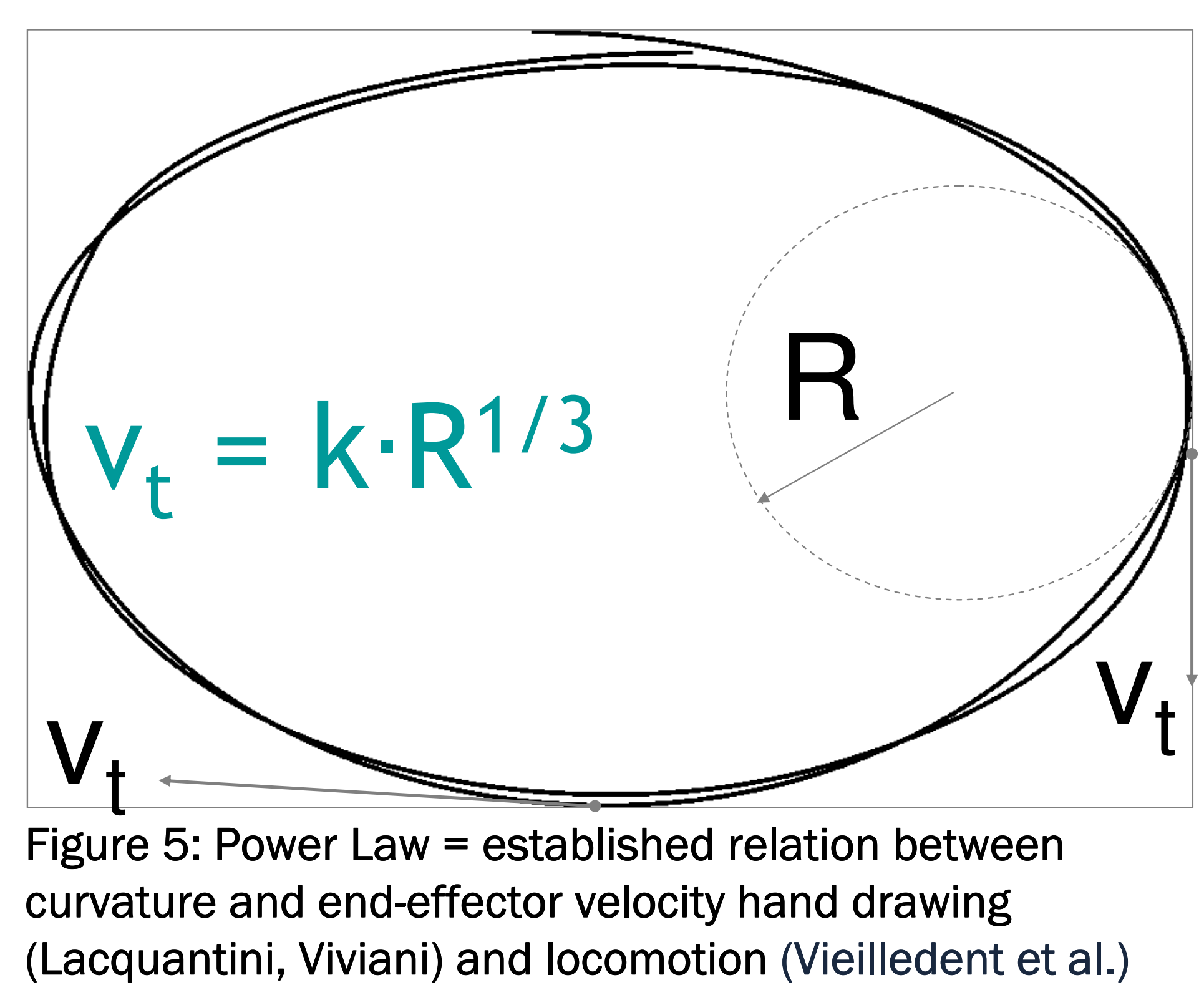

\section{Conclusion and Discussion:}

Leader's walking behavior is different from individual walking behavior but similar to the follower's walking behavior.

Because the leader is void of any task relevant visual information, it seems that the leader changes his behavior in the joint carrying condition based on task knowledge.

We did not find an indication for learning.

This work has been supported by the EU Project JAST. 